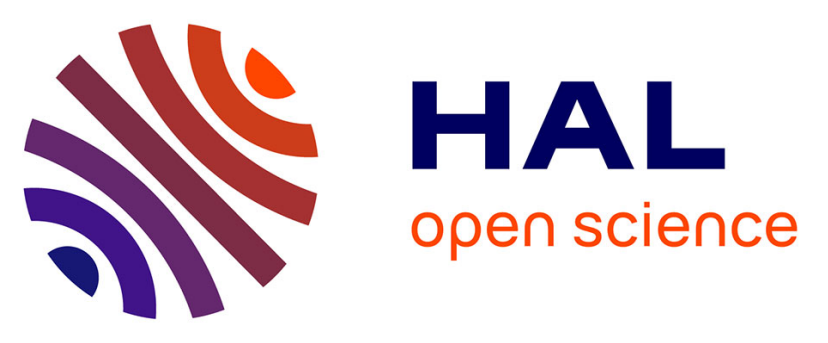

\title{
Coexistence of three predators competing for a single biotic resource
}

\author{
Claude Lobry, Tewfik Sari, Karim Yadi
}

\section{To cite this version:}

Claude Lobry, Tewfik Sari, Karim Yadi. Coexistence of three predators competing for a single biotic resource. Jean Lévine and Philippe Müllhaupt. Advances in the Theory of Control, Signals and Systems with Physical Modeling - Part III, Springer, pp.309-321, 2011, Lecture Notes in Control and Information Sciences - 407, 978-3-642-16134-6. 10.1007/978-3-642-16135-3_25. hal-00486459

\section{HAL Id: hal-00486459 \\ https://hal.science/hal-00486459}

Submitted on 25 May 2010

HAL is a multi-disciplinary open access archive for the deposit and dissemination of scientific research documents, whether they are published or not. The documents may come from teaching and research institutions in France or abroad, or from public or private research centers.
L'archive ouverte pluridisciplinaire HAL, est destinée au dépôt et à la diffusion de documents scientifiques de niveau recherche, publiés ou non, émanant des établissements d'enseignement et de recherche français ou étrangers, des laboratoires publics ou privés. 


\title{
COEXISTENCE OF THREE PREDATORS COMPETING FOR A SINGLE BIOTIC RESOURCE
}

\author{
CLAUDE LOBRY, TEWFIK SARI, AND KARIM YADI
}

\begin{abstract}
We construct a model of competition of three consumers for one single biotic resource ; simulations show that the three species coexist. Using singular perturbations theory we sketch a mathematical proof for this coexistence. The main mathematical tool used is an extension of the PontryaginRodygin theorem on the "slow" motion of a "slow-fast" differential system when the "fast" motion possesses a stable limit cycle. The mathematical analysis is done within the framework of Non Standard Analysis.
\end{abstract}

\section{INTRODUCTION}

The question of coexistence of competing species for a single resource has a very long history that we shall not attempt to recall here. We just recall the two decisive papers by Armstrong and Mac Gehee $[1,6]$ where they pointed that coexistence is not synonymous of coexistence at equilibrium. These papers were the starting point of numerous papers showing complex behaviors of systems of competitors and evidence of coexistence on the basis of numerical simulations. Following this tradition we propose a model of coexistence of three species competing for one resource.

The present paper has two parts. In the first part we construct our model and explain what is the rationale behind our construction ; each step is illustrated by simulations. In the second part we consider our model as a member of a more general "consumer-resource" model for which we explain how coexistence of species can be proved using singular perturbation analysis ; as an essential tool we use an extension of a theorem of Pontryagin and Rodygin.

Considered from the ecological point of view our model shows that oscillations in a "consumer-resource" relationship can open the door to coexistence with other species provided that the new introduced species do not perturb too much the oscillations. We do not know any example of an interaction between four species of the type of the model presented here but its existence is plausible. We shall explain it during the construction of a model. But we must acknowledge here that what we do is a kind of "virtual ecology" showing what is "theoretically possible" in a world of species respecting basic facts well established in concrete ecology. It is not a description of the real world!

From the mathematical point of view our paper can be considered as an application of singular perturbation methods to the mathematical proof of persistence for some specific system. Our contribution consists mainly in the analysis of the

2000 Mathematics Subject Classification. 92A15, 92A17, 34C15, 34C35, 34E18.

Key words and phrases. competition; coexistence; biotic resource; averaging; nonstandard analysis. 
theorem of Pontryagin and Rodygin and its extension to a theorem which is more effective in some circumstances. Detailed proofs can be consulted at [14] and will be published elsewhere. The mathematical analysis is done within the framework of Non Standard Analysis, using the axiomatic of Nelson [7] and respecting the spirit of G. Reeb [9].

\section{Construction of A model.}

2.1. The basic oscillating pair. We consider the system :

$$
\left\{\begin{aligned}
\frac{d s}{d t} & =3 s(1-s)-\frac{s^{2}}{0.01+s^{2}} x_{1} \\
\frac{d x_{1}}{d t} & =0.1\left[\frac{s^{2}}{0.01+s^{2}}-0.65\right] x_{1}
\end{aligned}\right.
$$

Due to the presence of the factor 0.1, it can be considered as a "slow-fast" system of two differential equations of the following type :

$$
\left\{\begin{aligned}
\frac{d s}{d t} & =\frac{1}{\varepsilon_{1}}\left[f(s)-g_{1}(s) x_{1}\right] \\
\frac{d x_{1}}{d t} & =\left(g_{1}(s)-d_{1}\right) x_{1}
\end{aligned}\right.
$$

where $\varepsilon_{1}$ is a "small" parameter. The real $s$ represents the density of some biotic resource (prey) for a consumer which density is represented by $x_{1}$. This is a rather classical prey-predator model and it is well known that this kind of generalization of the Lotka-Volterra model can have sustained oscillations. The existence of oscillations in prey-predator interaction is clearly demonstrated in laboratory experiments (see [4]), if not in the real world. The choice of the function :

$$
g_{1}(s)=\frac{s^{2}}{0.01+s^{2}}
$$

in place of the more classical Monod's function with $s$ in place of $s^{2}$ was made in order that the nullcline $\left[f(s)-g_{1}(s) x_{1}\right]=0$ has the "S-shape" shown on Fig. 1 which prevents the resource from extinction. This kind of assumption is sometimes called the "Allee" effect in ecological literature.

2.2. Addition of a new consumer " $x_{2}$ ". We want to add a new consumer and at the same time keep the oscillations of $\left(s, x_{1}\right)$. It can be done by introducing a new species with a very slow dynamics compare to that of $\left(s, x_{1}\right)$ like in the model :

where $\varepsilon_{2}$ is small.

$$
\left\{\begin{aligned}
\frac{d s}{d t} & =\frac{1}{\varepsilon_{1}}\left[f(s)-g_{1}(s) x_{1}-g_{2}(s) x_{2}\right] \\
\frac{d x_{1}}{d t} & =\left(g_{1}(s)-d_{1}\right) x_{1} \\
\frac{d x_{2}}{d t} & =\varepsilon_{2}\left(g_{2}(s)-d_{2}\right) x_{2}
\end{aligned}\right.
$$

The existence of two consumers (which densities are represented by $x_{1}$ and $x_{2}$ ) of the same resource and having very different characteristic time seems to be common in nature. For instance small mammals and big mammals eating the same grass have a lifespan which may differ of an order of magnitude. 


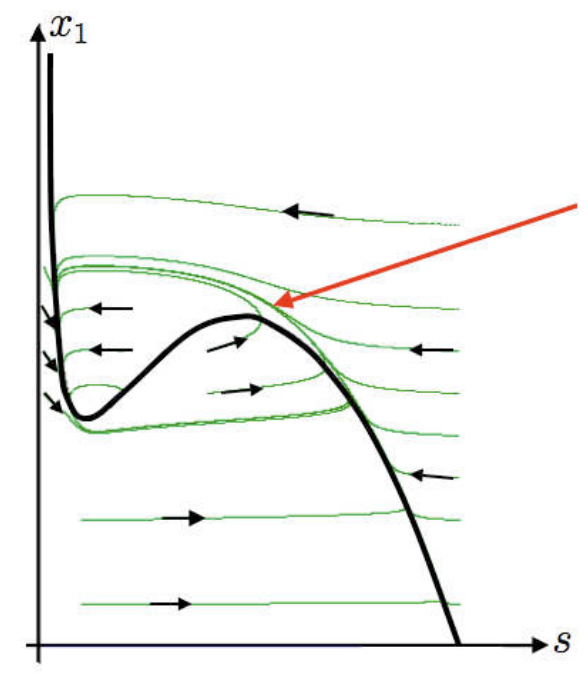

Figure 1. On this picture one observes a simulation of few trajectories of the system (1). The black "S-shaped" curve is the nullcline $\left[f(s)-g_{1}(s) x_{1}\right]=0$. The direction of the motion along trajectories is indicated by the black arrows and the limit cycle by the red arrow. By the way $s(t)$ oscillates between two values.

Denote by $\bar{x}_{2}$ some constant $x_{2}$; since $x_{2}(t)$ is quasi constant, for a while, the evolution of $\left(s, x_{1}\right)$ is governed by :

$$
\left\{\begin{array}{l}
\frac{d s}{d t}=\frac{1}{\varepsilon_{1}}\left[f(s)-g_{1}(s) x_{1}-g_{2}(s) \bar{x}_{2}\right] \\
\frac{d x_{1}}{d t}=\left(g_{1}(s)-d_{1}\right) x_{1}
\end{array}\right.
$$

In that system we see that when $\bar{x}_{2}$ is small the nullcline

$$
f(s)-g_{1}(s) x_{1}-g_{2}(s) \bar{x}_{2}=0
$$

is very close to the nullcline

$$
f(s)-g_{1}(s) x_{1}=0
$$

and, thus, oscillations are preserved. The range of oscillations of $s$ is slightly shortened as $\bar{x}_{2}$ increases. Now, if we look at the process from the point of view of $x_{2}$ during an oscillation of period $T$ the growth is given by :

$$
\int_{t}^{t+T}\left(g_{2}(s(\tau))-d_{2}\right) x_{2}(\tau) d \tau
$$

which varies monotonically according to the variation of amplitude of $s$. Thus the growth can be positive for small values of $x_{2}$ and negative for large ones; in the 


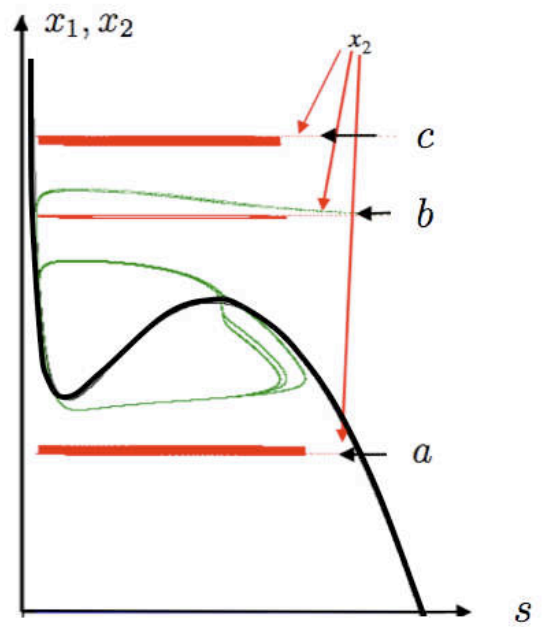

Figure 2. Simulation of the system (4). The picture shows the two projections of the trajectories, in green on the plane $\left(s, x_{1}\right)$ and in red on the plane $\left(s, x_{2}\right)$. While the initial conditions where kept constant for $s$ and $x_{1}$ they where changed for $x_{2}$. One sees that, starting from a small $x_{2}=a$ then $x_{2}(t)$ is increasing and, conversely, starting from a big $x_{2}=c$ then $x_{2}(t)$ is decreasing.

middle there must be an equilibrium. This is the case for the model :

$$
\left\{\begin{array}{l}
\frac{d s}{d t}=3 s(1-s)-\frac{s^{2}}{0.01+s^{2}} x_{1}-g_{2}(s) x_{2} \\
\frac{d x_{1}}{d t}=0.1\left[\frac{s^{2}}{0.01+s^{2}}-0.65\right] x_{1} \\
\frac{d x_{2}}{d t}=0.01\left[g_{2}(s)-0.025\right] x_{2}
\end{array}\right.
$$

with

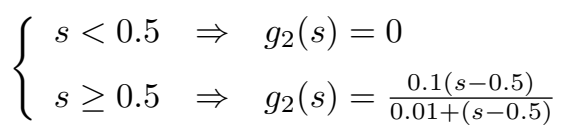

We choose $g_{2}=0$ on $[0,0.5]$ in order to be sure that the nullcline (2) is exactly the same than the nullcline (3) and remains "S-shaped" ; thus oscillations are preserved. This artificial choice is convenient for simplicity but a model with $g_{2}$ being smoother would also work. Evidence of coexistence of $x_{1}$ and $x_{2}$ is given on Fig. 2. On this simulation three initial conditions where taken, keeping $s(0)$ and $x_{1}(0)$ constant and changing $x_{2}(0)$. On the picture we have superimposed the projections on the $\left(s, x_{1}\right)$ plane (in green) and the $\left(s, x_{2}\right)$ plane in red. The first initial condition for $x_{3}$ is $a$ which is shown by the black arrow ; since the variation of $x_{2}$ is very slow the red projection looks like a point moving right to left and left to right very fast while moving up very slowly ; the thick red line corresponds to a dozen of oscillations. Starting from $c$ we see that the point is slowly moving down. Starting from $b$ we see that $x_{2}$ remains constant.

2.3. Addition of a new consumer " $x_{3}$ ". The idea is to have a new consumer with a $g_{3}$ growth rate such that the two graphs of $g_{2}$ and $g_{3}$ cross like on Fig. 3 


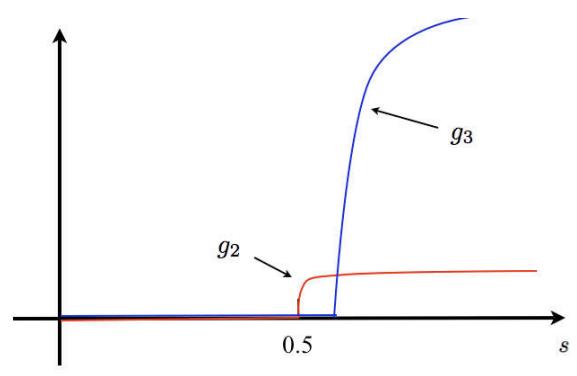

Figure 3 . The graphs of $g_{2}$ and $g_{3}$.

in order that during an oscillation of $\left(s, x_{1}\right)$ the species 2 and 3 take the advantage alternatively. This leads to the system :

$$
\left\{\begin{aligned}
\frac{d s}{d t} & =3 s(1-s)-\frac{s^{2}}{0.01+s^{2}} x_{1}-g_{3}(s) x_{3} \\
\frac{d x_{1}}{d t} & =0.1\left[\frac{s^{2}}{0.01+s^{2}}-0.65\right] x_{1} \\
\frac{d x_{3}}{d t} & =0.01\left[g_{3}(s)-0.025\right] x_{3}
\end{aligned}\right.
$$

with

$$
\left\{\begin{array}{lll}
s<0.58 & \Rightarrow & g_{3}(s)=0 \\
s \geq 0.58 & \Rightarrow & g_{3}(s)=\frac{2(s-0.58)}{0.01+(s-0.58)}
\end{array}\right.
$$

On Fig. 4 one sees that the behavior of the system $\left(s, x_{1}, x_{3}\right)$ is similar to the behavior we observed for $\left(s, x_{1}, x_{2}\right)$; the projection (in blue) on the $\left(s, x_{3}\right)$ plane is similar to the projection (in red) observed for $\left(s, x_{2}\right)$ in Fig.2.

2.4. Coexistence of all the three consumers. Now we consider the complete system :

$$
\left\{\begin{aligned}
\frac{d s}{d t} & =3 s(1-s)-\frac{s^{2}}{0.01+s^{2}} x_{1}-g_{2}(s) x_{2}-g_{3}(s) x_{3} \\
\frac{d x_{1}}{d t} & =0.1\left[\frac{s^{2}}{0.01+s^{2}}-0.65\right] x_{1} \\
\frac{d x_{2}}{d t} & =0.01\left[g_{2}(s)-0.025\right] x_{2} \\
\frac{d x_{3}}{d t} & =0.01\left[g_{3}(s)-0.025\right] x_{3}
\end{aligned}\right.
$$

Species $x_{2}$ and $x_{3}$ have a "slow motion" which can be approximated by computing suitable integrals along the basic cycle; this determines a flow on $\left(x_{2}, x_{3}\right)$ plane ; this flow is studied and proved to have a stable equilibrium which proves the persistence of both $x_{2}$ and $x_{3}$. On Fig. 5 one sees the projection on the $\left(x_{2}, x_{3}\right)$ plane of the full system (6) with the three competitors. The simulations from various initial conditions shows convergence to a point which actually corresponds to a periodic orbit in the full space $\left(s, x_{1}, x_{2}, x_{3}\right)$. More details are given in the next section.

2.5. Species 2 and 3 alone. Consider the system with $s, x_{2}$ and $x_{3}$ alone in the absence of the species represented by $x_{1}$. One easily check that in this case, from the choice of $g_{2}$ and $g_{3}$, there is no oscillation and the stable equilibrium is the one for which species $x_{2}$ wins the competition. 


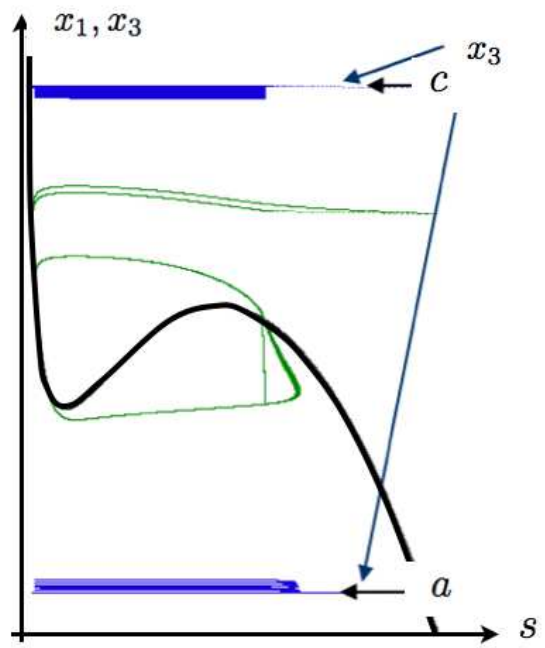

Figure 4. Simulation of the system (5). The picture shows the two projections of the trajectories, in green on the plane $\left(s, x_{1}\right)$ and in blue on the plane $\left(s, x_{3}\right)$. The projection (in blue) on the $\left(s, x_{3}\right)$ plane is similar to the projection (in red) observed for $\left(s, x_{2}\right)$ in Fig.2. Starting from a small $x_{3}=a$ then $x_{3}(t)$ is increasing and, conversely, starting from a big $x_{3}=c$ then $x_{3}(t)$ is decreasing. Between $a$ and $c$ there is some initial condition (not represented) for which $x_{3}$ do not increase nor decrease.

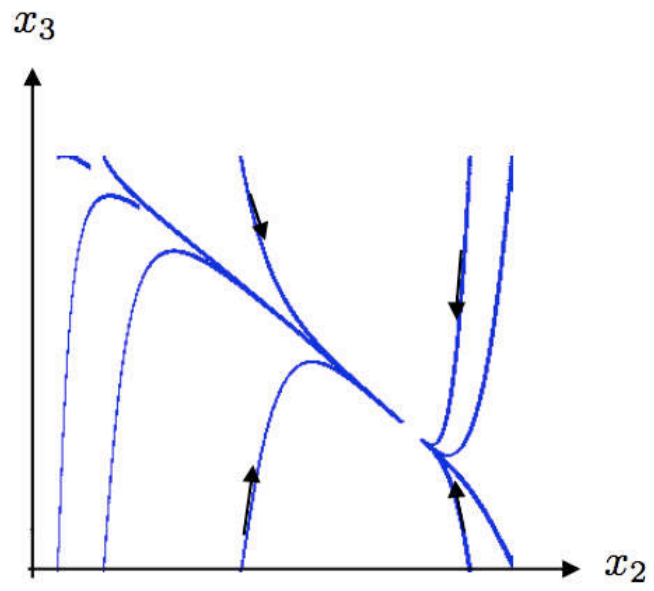

FiguRE 5. On this picture we have represented the projection of simulations of the the complete system (6) on the plane $\left(x_{2}, x_{3}\right)$. The variables $\left(s_{1}, x_{1}\right)$ (not represented) present rapid oscillations while $\left(x_{2}, x_{3}\right)$ evolves slowly. One sees that all the trajectories seem to converge to an equilibrium. Compare to the "theoretical" picture on Fig 8. 


\section{Proof of the Persistence of the three COMPetitors in A MOdel WITH THREE TIME SCALES}

In this section, we give the successive steps of a proof of the persistence in a model of the form

$$
\left\{\begin{aligned}
\frac{d s}{d t} & =\frac{1}{\varepsilon_{1} \varepsilon_{2}}\left(f(s)-g_{1}(s) x_{1}-g_{2}(s) x_{2}-g_{3}(s) x_{3}\right) \\
\frac{d x_{1}}{d t} & =\frac{1}{\varepsilon_{1}}\left(g_{1}(s)-d_{1}\right) x_{1} \\
\frac{d x_{2}}{d t} & =\left(g_{2}(s)-d_{2}\right) x_{2} \\
\frac{d x_{3}}{d t} & =\left(g_{3}(s)-d_{3}\right) x_{3}
\end{aligned}\right.
$$

where the occurring functions are differentiable, at least piecewise, $\varepsilon_{1}$ and $\varepsilon_{2}$ are infinitesimal $^{1}$. The function $f$ vanishes at 0 ; it is increasing then decreasing and vanishes at a value $m$. The functions $g_{i}$ are zero at 0 , increasing and bounded.

3.1. Oscillations of $s$ and $x_{1}$. Let us consider the system

$$
\left\{\begin{aligned}
\frac{d s}{d t} & =\frac{1}{\varepsilon_{1}}\left(f(s)-g_{1}(s) x_{1}\right) \\
\frac{d x_{1}}{d t} & =\left(g_{1}(s)-d_{1}\right) x_{1}
\end{aligned}\right.
$$

- Suppose that the nullcline $d s / d t=0$ is a curve $\varphi$ that, when $s$ increases from 0 , decreases from $+\infty$ to a minimum value reached for $s=s^{-}$then increases to a maximum value for $s=s^{+}$to finally decrease and vanishes for $s=m$.

- Suppose that the value $s^{*}$ such that $g_{1}\left(s^{*}\right)=d_{1}$ is between $s^{-}$and $s^{+}$.

Proposition 3.1. For $\varepsilon_{1}$ infinitesimal, the system (8) has a limit cycle close to the curve $A B C D$ in Fig. 6.

3.2. The Pontryagin-Rodygin's Theorem. Due to the lack of space, we shall try in these sections to avoid excessive mathematical formalism of the results and we refer to $[12,14,13]$ for more details. To simplify, we suppose that all the occurring differential equations have the property of uniqueness of solutions. Let us consider the slow and fast system

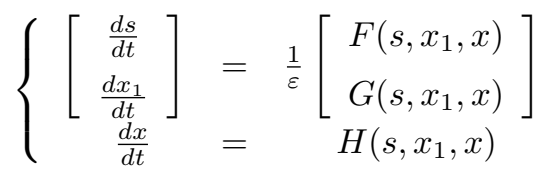

where the scalars $s$ and $x_{1}$ are the fast components, and the vector $x$ the slow one. The real number $\varepsilon$ is positive and infinitesimal. The functions $F, G$ and $H$ are continuous. The following system, where $x$ is considered as a parameter, is called the fast equation

$$
\left[\begin{array}{c}
\frac{d s}{d t} \\
\frac{d x_{1}}{d t}
\end{array}\right]=\frac{1}{\varepsilon}\left[\begin{array}{c}
F\left(s, x_{1}, x\right) \\
G\left(s, x_{1}, x\right)
\end{array}\right]
$$

Hence, the $\left(s, x_{1}\right)$-component of a solution of $(9)$ varies very quickly according to (10) where $x$ has been frozen at its initial value. When the fast equation (10) has stable limit cycles $\Gamma_{x}$ for each $x$ in a compact domain, Pontryagin-Rodygin's

\footnotetext{
${ }^{1}$ We use N.S.A. terminology. Following the spirit of ([5]) when a word (like "infinitesimal") is written in bold character its meaning is the one used in the formal language of Nelson I.S.T. but the reader not familiar with this framework can use the intuitive meaning.
} 


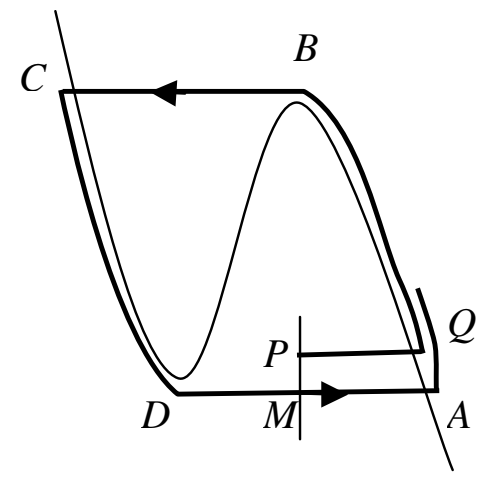

Figure 6. Starting from the point $P$ the solution is quasi horizontal and goes fast to the nullcline $d s / d t=0$; then the solution stays near the nullcline and goes up until it reaches the maximum at $B$; then the solution is quasi horizontal and goes fast to the nullcline $d s / d t=0$ at point $C$; then the solution stays near the nullcline and goes down until it reaches the minimum at $D$; then the solution goes fast to the right to the nullcline $d s / d t=0$ and reaches it at $A$.

Theorem [8] gives the limiting behavior of the singularly perturbed problem (9): Under suitable conditions, after a fast transition near the cycles described by the fast equation (10), the trajectories of (9) quickly roll up around the manifold generated by the cycles, with a slow evolution of the x-component according to the averaged system

$$
\frac{d x}{d t}=\frac{1}{P(x)} \int_{0}^{P(x)} H\left(s^{*}(\tau, x), x_{1}^{*}(\tau, x), x\right) d \tau
$$

where $\left(s^{*}(\tau, x), x_{1}^{*}(\tau, x)\right)$ is a $P(x)$-periodic solution of the fast equation corresponding to the cycle $\Gamma_{x}$. This result was originally obtained for at least $C^{2}$ vector fields, under the assumption that the cycles $\Gamma_{x}$ are asymptotically stable in the linear approximation. However, the result obtained in [12] shows that PontryaginRodygin description of solutions holds for $C^{0}$ vector fields under additional assumptions.

3.3. Extension of Pontryagin-Rodygin's Theorem. Note that System (7) has the form

$$
\left\{\begin{array}{c}
{\left[\begin{array}{c}
\frac{d s}{d t} \\
\frac{d x_{1}}{d t}
\end{array}\right]=\frac{1}{\varepsilon_{2}}\left[\begin{array}{c}
\frac{1}{\varepsilon_{1}} F\left(s, x_{1}, x\right) \\
G\left(s, x_{1}, x\right) \\
\frac{d x}{d t}
\end{array}\right]=\quad H\left(s, x_{1}, x\right)}
\end{array}\right.
$$

where $x=\left(x_{2}, x_{3}\right) \in \mathbb{R}^{2}$. The fast equation

$$
\left[\begin{array}{c}
\frac{d s}{d t} \\
\frac{d x_{1}}{d t}
\end{array}\right]=\frac{1}{\varepsilon_{2}}\left[\begin{array}{r}
\frac{1}{\varepsilon_{1}} F\left(s, x_{1}, x\right) \\
G\left(s, x_{1}, x\right)
\end{array}\right]
$$




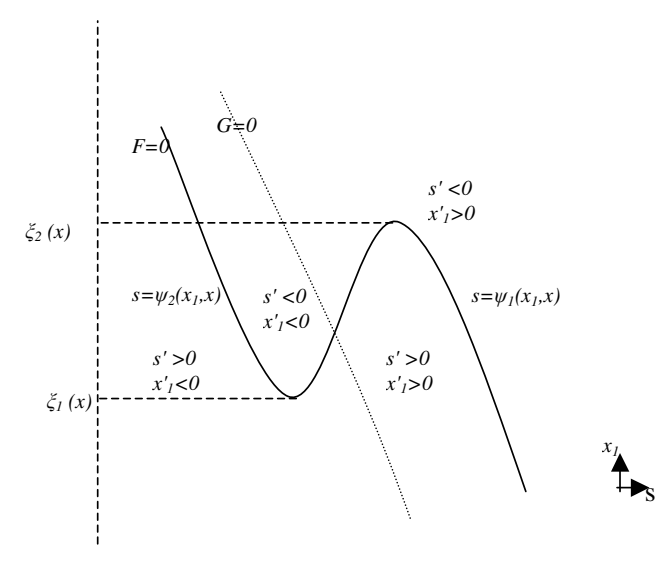

Figure 7. Notations in equations (14)

admits a stable limit cycle for any value of $x$ for infinitesimal values of $\varepsilon_{1}$. It is tempting to apply Pontryagin-Rodygin's Theorem to (12) but the main reason that makes this impossible is the fact that the fast equation is a nonstandard equation. It is itself a singularly perturbed equation. We can not avoid to take into account the three dynamics of the problem. In [14] Pontryagin-Rodygin's Theorem is extended to this kind of system the fast equation of which admits a slow and fast limit cycle. This new result has also the advantage to overcome a serious limitation of Pontryagin-Rodygin's Theorem : unlike the latter, it makes possible the localization of the cycles, the approximation of their periods and the calculation of the average along these cycles. The functions $F, G$ and $H$ being continuous and the positive real numbers $\varepsilon_{1}$ and $\varepsilon_{2}$ infinitesimal, suppose that there exists a compact domain $K$ of $\mathbb{R}^{2}$ such that, for all $x \in K$ the nullclines $F=0$ and $G=0$ of (13) have the shape given in Figure 7 . The $\left(s, x_{1}\right)$-plane is divided in four regions where the field has the indicated signs in the figure. The limit cycle of (13) is infinitesimally close to the closed curve $(A B C D)$ in Fig. 6 formed by two "slow arcs" $(A B)$ and $(C D)$ and two "fast segments" $(D A)$ and $(B C)$. The two decreasing branches of the nullcline $F=0$ are denoted $s=\psi_{1}\left(x_{1}, x\right)$ and $s=\psi_{2}\left(x_{1}, x\right)$. Let us define in the interior of $K$ the slow equation

$$
\frac{d x}{d t}=M(x)
$$

where

$$
\begin{gathered}
M(x)=\frac{1}{P(x)} \sum_{i=1}^{2} \int_{\xi_{i}(x)}^{\xi_{i+1}(x)} \frac{g\left(\psi_{i}\left(x_{1}, x\right), x_{1}, x\right)}{f_{2}\left(\psi_{i}\left(x_{1}, x\right), x_{1}, x\right)} d x_{1}, \\
P(x)=\sum_{i=1}^{2} \int_{\xi_{i}(x)}^{\xi_{i+1}(x)} \frac{d x_{1}}{f_{2}\left(\psi_{i}\left(x_{1}, x\right), x_{1}, x\right)} \text {, with } \xi_{3}(x)=\xi_{1}(x) .
\end{gathered}
$$

Let $\gamma(t)$ be the trajectory of a solution of (12). Theorem 5.2.1 page 75 in [14] 


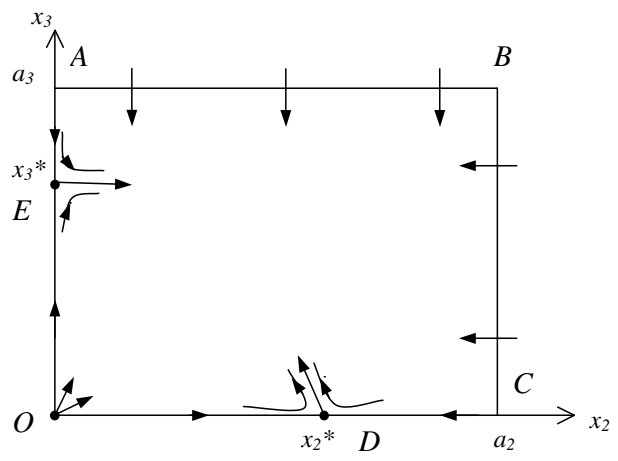

Figure 8. Portrait of $\left(x_{2}, x_{3}\right)$ obtained from the averaged system (16). Compare to the simulations presented on Fig.5

explains how $\gamma(t)$ behave in the same manner than in the classical PontryaginRodygin theorem, the averaging on the cycles being now well approximated by the explicit formulas (15).

3.4. Application to the model. Reconsider System (7).

- Assume that the functions $g_{2}$ and $g_{3}$ are zero until the respective thresholds $s_{2}$ and $s_{3}$ are reached such that $\min \left(s_{2}, s_{3}\right) \geq s^{+}$and that $g_{2}$ and $g_{3}$ are increasing beyond.

This assumption allows us to assert that the subsystem

$$
\left[\begin{array}{c}
\frac{d s}{d t} \\
\frac{d x_{1}}{d t}
\end{array}\right]=\frac{1}{\varepsilon_{2}}\left[\begin{array}{l}
\frac{1}{\varepsilon_{1}}\left(f(s)-g_{1}(s) x_{1}-g_{2}(s) x_{2}-g_{3}(s) x_{3}\right) \\
\left(g_{1}(s)-d_{1}\right) x_{1}
\end{array}\right]
$$

still admits, for every $\left(x_{2}, x_{3}\right)$ and $\varepsilon_{1}$ small enough, a limit cycle $\Gamma_{x_{2}, x_{3}}$ that differs from that of (8) for values of $s \geq \min \left(s_{2}, s_{3}\right)$. The more $x_{1}$ and $x_{2}$ are large, the more these cycles are distorted inwards in their right side. The minimum and maximum of the cycles remain unchanged. Here, the averaged equation $(14,15)$ takes the form (see [14] for explicit formulas)

$$
\left\{\begin{array}{l}
d x_{2} / d t=x_{2} M_{2}\left(x_{2}, x_{3}\right) / P\left(x_{2}, x_{3}\right), \\
d x_{3} / d t=x_{3} M_{3}\left(x_{2}, x_{3}\right) / P\left(x_{2}, x_{3}\right) .
\end{array}\right.
$$

A detailed study of equation (16) leads to the following conditions of persistence :

Theorem 3.2. [14] Suppose that $s_{3}>s_{2}=s^{+}, \varphi\left(s_{3}\right)>\varphi\left(s^{-}\right)$and that $d_{2}$ is below a certain constant well determined by the problem. Then, for $s_{3}-s_{2}$ and $d_{3}$ small enough, there is persistence of the species $x_{2}$ and $x_{3}$ of (16).

This result is reflected in Fig. 8 representing a positively invariant box $O A B C$ of (16) in which arrive all trajectories with positive initial conditions. The axes are invariant, the origin $O$ is an unstable node and $C$ and $E$ are saddle points. A lemma due to Butler-McGehee [2] shows that the union of limit sets of positive half-trajectories is a compact subset $\Omega$ which does not meet the axes.

Theorem 3.3. Under the assumptions of the preceding Theorem, there is persistence of the whole species of the model (7) for all positive initial conditions. 
This final result is obtained by using a nonstandard permanence lemma [10] which extends the approximation of the component $\left(x_{2}(t), x_{3}(t)\right)$ of the solution of (7) by the solution of (16) to an infinitely large time interval $[0, \omega]$. We than prove $^{2}$ that $\left(x_{2}(t), x_{3}(t)\right)$ remains infinitely close to $\Omega$ for all infinitely large values of $t$ and all infinitesimal values of $\varepsilon_{1}$ and $\varepsilon_{2}$.

\section{REFERENCES}

[1] R. A. Armstrong, R. McGehee. Coexistence of species competing for shared resources. Theoretical Pop. Biol.,(9) : 317-328,1976.

[2] G. Butler, P. Waltman. Persistence in dynamical systems. J. Differential Equations, (63) : 255-263, 1986.

[3] J-L. Callot, T. SARI. Stroboscopie et moyennisation dans les systèmes d'equations diff'erentielles à solutions rapidement oscillantes. Mathematical Tools and Models for Control, Systems Analysis and Signal Processing, CNRS Paris, (3) : 345-353, 1983.

[4] J.L. Jost, T. J.F. Drake, A.G. Frederickson, M. Tsuchiya. Interaction of Tetrahimen pyriformis, Escherichi coli, Azotobacter vinelandi and Glucose in a Minimal Madium. J. of Bacteriology, 113(2) : 834-840, 1973.

[5] C. Lobry, T. SARI. Nonstandard analysis and representation of real world. International Journal on Control, 80(3) : 171-193, 2007.

[6] R. McGehee, R. A. Armstrong. Some mathematical problems concerning the ecological principle of competitive exclusion. Journal od Differential Equations, (23) : 30-52, 1977.

[7] E. Nelson. Internal Set Theory: a new approach to nonstandard analysis. Bull. Amer. Math. Soc., 83 (6) : 1165-1198, 1977.

[8] L. S. Pontryagin, L. V. Rodygin. Approximate solution of a system of ordinary differential equations involving a small parameter in the derivatives. Soviet. Math. Dokl., (1) : 237-240, 1960.

[9] G. Reeb. La mathématique non standard vieille de soixante ans ? Troisième Colloque sur les Catégories, dédié à C. Ehresmann, Amiens, 1980. Cahiers Topologie Géom. Différentielle 22(2):149-154, 1981.

[10] A. Robinson. Nonstandard Analysis. American Elsevier, New York (1974).

[11] T. SARI. Averaging in Ordinary Differential Equations and Functional Differential Equations, The Strength of Nonstandard Analysis. I. van den Berg, V. Neves (editors). Springer-Verlag, Wien, 286-305,2007.

[12] T. SARI, K. YAdi. On Pontryagin-Rodygin's theorem for convergence of solutions of slow and fast systems. Electron. J. Diff. Eqns, 2004 (139) : 1-17,2004.

[13] K. YADI. Singular perturbations on infinite time interval. Revue Arima, Vol. 9 (2008), 37-560.

[14] K. YAdi. Perturbations Singulières : Approximations, Stabilité Pratique et Applications à des Modèles de Compétition. Thèse de doctorat de l'Université de Haute-Alsace de Mulhouse, 2008. http://tel.archives-ouvertes.fr/tel-00411503/fr/

C. Lobry, EPI-MERE, INRIA-Sophia Antipolis, FR.

E-mail address: claude.lobry@inria.fr

T. Sari, Laboratoire de Mathmatiques, Informatique et Applications, Universite de Haute Alsace, 4 Rue des frères Lumière, 68093 Mulhouse, and EPI MERE (INRIA-INRA),

UMR MisteA, INRA 2, Pl. Viala, 34060 Montpellier, France.

E-mail address: Tewfik.Sari@uha.fr

K. Yadi, Dynamical systems and Applications Laboratory, University Aboubekr Belkaïd, TLemCen, DZ.

E-mail address: k.yadi@mail.univ-tlemcen.dz

\footnotetext{
${ }^{2}$ We say that the standard set $\left\{\Gamma_{x_{2}, x_{3}} \times\left\{\left(x_{2}, x_{3}\right)\right\}:\left(x_{2}, x_{3}\right) \in \Omega\right\}$ is practically asymptotically stable for (7) for all infinitesimal values of $\varepsilon_{1}$ and $\varepsilon_{2}$ (see [13] for more details).
} 